\title{
Strong Decays: Past, Present and Future
}

\author{
T.Barnes \\ Department of Physics, University of Tennessee, Knoxville, TN 37996, USA \\ Physics Division, Oak Ridge National Laboratory, Oak Ridge, TN 37831, USA
}

\begin{abstract}
In this talk I review the history of models of strong decays, from the original model through applications to charmonium, light and charmed mesons, glueballs and hybrids. Our current rather limited understanding of the QCD mechanism of strong decays is stressed. Regarding current and future applications of strong decay models, we note that in certain channels the very strong coupling predicted between $q \bar{q}$ basis states and the two-meson continuum may lead to strongly mixed states and perhaps molecular two-meson bound states. The relevance to the $\mathrm{D}_{s J}^{*}(2317)$ is discussed.
\end{abstract}

\section{HISTORICAL INTRODUCTION}

\section{Origins of the ${ }^{3} \mathrm{P}_{0}$ model}

\section{Micu}

The earliest reference in which the currently widely accepted microscopic physics of strong decays appears is due to Micu [1], in the paper "Decay Rates of Meson Resonances in a Quark Model". Micu was concerned with understanding the light P-wave mesons in the quark model, especially their widths. In the quark model, describing decays required the production of a $q \bar{q}$ pair; in lieu of a microscopic model of interactions between quarks she made the plausible assumption that the pair was produced with vacuum $\left(0^{++}\right)$quantum numbers, therefore in a ${ }^{3} \mathrm{P}_{0}$ state. No explicit quark model wavefunctions were assumed, so the implicit overlap integrals were described by two free parameters, taken from data. Micu applied this simple model to approximately 30 known light meson decays, and found that it was reasonably successful in explaining the observed partial widths. She concluded that "... this decay model proves once more the surprising viability of the quark model."

\section{The ORSAY group}

This work was follows by a series of decay model calculations by the ORSAY group (LeYaouanc et al.), who introduced explicit nonrelativistic quark model wavefunctions in the calculations of the decay amplitudes and a fundamental pair production amplitude $\gamma$. This cast the ${ }^{3} \mathrm{P}_{0}$ decay model in essentially the form in which it is used today. 
In their initial 1973 paper [2] the ORSAY group set up the formalism of ${ }^{3} \mathrm{P}_{0}$ decays in the quark model and applied it to light baryon and meson decays and couplings. The importance of the $\mathrm{D} / \mathrm{S}$ amplitude ratios in the decays $b_{1} \rightarrow \omega \pi$ and $a_{1} \rightarrow \rho \pi$ as crucial tests of the assumed ${ }^{3} \mathrm{P}_{0}$ quantum numbers of the $q \bar{q}$ pair was first stressed in this paper; this early and striking success of the ${ }^{3} \mathrm{P}_{0}$ model was strong evidence in favor of this model of strong decays. This paper also notes that the model predicts strong threemeson effective couplings and form factors, which is an application that has not yet been widely exploited, but is of great importance for the currently fashionable meson effective lagrangians and meson exchange models of hadronic reactions.

This introductory paper was followed by detailed studies of baryon decays [3, 4], which considered $\approx 100$ baryon decay amplitudes. Finally, with the discovery of charmonium and states above open-charm threshold, the open-charm decays of charmonia were considered [5], and it was noted that the $\psi(4040)$ (elsewhere suggested as a $\mathrm{D}^{*} \mathrm{D}^{*}$ molecule candidate due to its anomalous strong branching fractions) could be accepted as a conventional $3{ }^{3} \mathrm{~S}_{1} c \bar{c}$ state, since the nodes of the radial wavefunction could plausibly weaken the DD and DD* modes.

It is straightforward to give Feynman rules for this ${ }^{3} \mathrm{P}_{0}$ model, since as noted by Ackleh et al. [6] it corresponds to the nonrelativistic limit of a $\bar{\psi} \psi$ decay interaction. The two diagrams that describe $q \bar{q}$ decays are shown in Fig. 1 below.
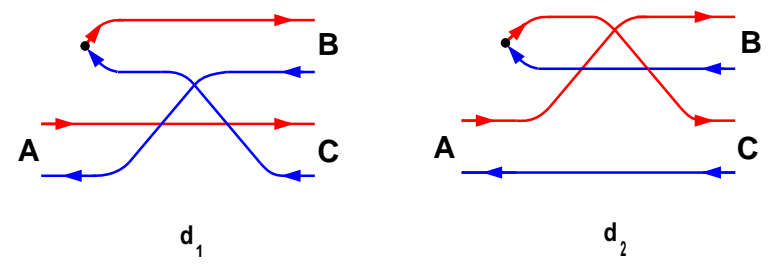

FIGURE 1. The two independent meson decay diagrams in the ${ }^{3} \mathrm{P}_{0}$ model.

\section{The Cornell charmonium decay model}

The discovery of charmonium motivated the next important development in decay models. This was the introduction of a new microscopic model for the QCD mechanism underlying strong decays, as well as studies of coupled-channels effects, in a series of papers by the Cornell group [7, 8, 9].

Eichten et al. assumed that strong decays were driven by $q \bar{q}$ pair production from the linear confining interaction. This implied that the size of the phenomenological $q \bar{q}$ pair production amplitude $\gamma$ in the ${ }^{3} \mathrm{P}_{0}$ model was proportional to the $q \bar{q}$ string tension, to the extent that these models could be compared.

A crucial difference between the Cornell model and most other decay and confinement models was their assumption of timelike vector confinement. This was subsequently rejected due to its inaccuracy in describing the splittings of the P-wave $\chi_{J}$ multiplet; vector confinement gives a positive spin-orbit term that does not agree with the data, 


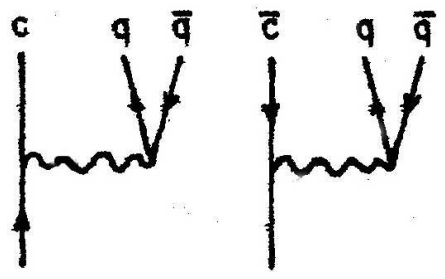

FIGURE 2. The Cornell decay model: pair production from linear vector confinement.

whereas scalar confinement has negative spin-orbit that leads to a good description of the $\chi_{J}$ masses. This issue is certainly not settled, and recent theoretical work by Swanson and Szczepaniak again argues in favor of vector confinement models [10].

The Cornell group did not give individual decay amplitudes and partial widths for resonances above open-charm threshold, but instead used a "resolvant" formalism to infer their combined contribution to $R$. This gave results that are quite similar to the current experimental $R$, and (even if this model of confinement proves incorrect) shows that this decay model merits further study.

Finally, the very topical issue of the level of mixing between "bare" $c \bar{c}$ basis states and the two-meson continuum was also considered by the Cornell group. They found that this is a significant effect, with (in their final version of this model [9]) the 1P $\chi_{J}$ states being $\approx 10 \%$ meson-meson and the $2 \mathrm{~S} \psi^{\prime} \approx 20 \%$ meson-meson. They warn however that their two-meson intermediate states are truncated at $\mathrm{DD}^{*}$, so the actual two-meson component may be much larger.

\section{MODERN "SURVEYS" OF DECAY MODES}

\section{$\xi(2230)$ brou ha ha}

Most of the detailed "survey" studies of strong decays that have appeared since 1980 have assumed the ${ }^{3} \mathrm{P}_{0}$ model, either as originally formulated or in one of several variants that modify the phase space, kinematics or the spatial dependence of the pair production amplitude. These survey papers typically attempt to consider as many open-flavor decay modes as possible, due to the "brou ha ha" associated with the question of the $\xi(2230)$.

The $\xi(2230)$ was originally reported in $J / \psi$ radiative decays by MarkIII at SLAC [11] in KK final states (apparently both charged and neutral kaons). The state appeared remarkably narrow, hence it was considered a possible glueball candidate. However there were problems with confirmation of the $\xi(2230)$; DM2 for example did not see it, and they had slightly better statistics than Mark III. For those with long memories, the reports of the $\Theta(1542)$ at this meeting are disturbingly reminiscent of the $\xi(2230)$.

Although the narrow width of the $\xi(2230)$ made it a plausible tensor glueball candidate, assuming that it did exist, this exciting possibility was dampened by a "spoiler" paper by Godfrey et al. [12], who noted that $\mathrm{L}=3 s \bar{s}$ states were expected near this mass, 
and they should "accidentally" be quite narrow (due to limited phase space and centrifical barriers), provided that their decays were dominated by decays to two S-wave mesons $\left(\mathrm{KK}, \mathrm{KK}^{*}\right.$ and $\mathrm{K}^{*} \mathrm{~K}^{*}$ ). The ${ }^{3} \mathrm{~F}_{2} s \bar{s}$ assignment was preferred because $g g$ intermediate states in $J / \psi$ radiative decays were predicted to populate $\mathrm{J}^{\mathrm{PC}}=0^{ \pm+}$and $2^{++}$ final states preferentially.

This a priori plausible assumption of dominant decays to S-wave meson pairs was subsequently tested by Godfrey et al. [13], who carried out a more complete set of decay calculations. They were surprised to find that the decays of a ${ }^{3} \mathrm{~F}_{2} s \bar{s}$ meson were not dominated by $\mathrm{S}$-wave meson pair final states. Instead the dominant mode was the $\mathrm{S}+\mathrm{P}$ final state $\mathrm{KK}_{1}(1270)$ by a considerable margin, so this $s \bar{s}$ state was instead predicted to be rather broad, once all the higher modes were included. Thus the $\xi(2230)$ could no longer be claimed to be an accidentally narrow ${ }^{3} \mathrm{~F}_{2} s \bar{s}$ state.

This specific result has broad implications. Evidently one should be cautious in using strong decay calculations to estimate total widths; calculations of all open-flavor decay amplitudes and partial widths are prudent. The assumption of dominance by a specific set of low-lying S-wave final mesons may well be inaccurate. For this reason, several recent papers on strong decays of light mesons have given results for all allowed openflavor modes, and this approach should probably be followed in future studies of baryon and heavy meson strong decays as well.

\section{Recent decay surveys}

The past two decades have seen a series of detailed calculations of strong decay amplitudes and partial widths using the ${ }^{3} \mathrm{P}_{0}$ model or a variant. For mesons the best known is the encyclopaedic work by Godfrey and Isgur [14], and for baryons the corresponding paper by Capstick and Isgur [15]. These studies have been followed by more complete studies of decay modes. The paper of Barnes, Close, Page and Swanson [16] evaluates all open two-body modes of all nonstrange mesons expected in the quark model to $2.1 \mathrm{GeV}$; this was recently extended to all open modes of strange mesons in the same multiplets by Barnes, Black and Page [17]. Calculations of nonstrange baryon decays were extended to include modes with vector mesons by Capstick and Roberts [18]; an extensive review of baryon strong decays was recently published by the same authors [19].

\section{New results for decays of strange mesons}

As this is a conference that is largely devoted to the physics of mesons, it may be of interest to review some of the recent theoretical results on strange meson decays [17]. First, regarding the axial vectors, from the very broad width predicted for a $1^{++}$state at the mass of the $f_{2}^{\prime}(1525)$ (ca. $400 \mathrm{MeV}$ ) it is clear that there is no "hidden" axial vector state with the same mass and width as the $f_{2}^{\prime}$. The near-threshold $f_{1}(1420)$ does appear consistent with the predicted width, although the nearby $\mathrm{KK}^{*}$ threshold will modify the 
shape of this resonance. Second, one often hears the speculation that $s \bar{s}$ mesons might be rather narrow, and hence offer attractive experimental targets. In our survey the five narrowest $s \bar{s}$ states we found without widely accepted experimental candidates were

${ }^{1} \mathrm{D}_{2} \eta_{2}(1850), \Gamma=129 \mathrm{MeV}$, dominant mode $\mathrm{KK}^{*}$

${ }^{3} \mathrm{~F}_{4} f_{4}(2200), \Gamma=156 \mathrm{MeV}$, dominant modes $\mathrm{KK}, \mathrm{KK}^{*}, \mathrm{~K}^{*} \mathrm{~K}^{*}$

$3{ }^{1} \mathrm{~S}_{0} \eta(1950), \Gamma=175 \mathrm{MeV}$, dominant modes $\mathrm{KK}^{*}, \mathrm{~K}^{*} \mathrm{~K}^{*}$

$2^{1} \mathrm{P}_{1} h_{1}(1850), \Gamma=193 \mathrm{MeV}$, dominant modes $\mathrm{KK}^{*}, \mathrm{~K}^{*} \mathrm{~K}^{*}, \eta \phi$

${ }^{3} \mathrm{D}_{2} \phi_{2}(1850), \Gamma=214 \mathrm{MeV}$, dominant modes $\mathrm{KK}^{*}, \eta \phi$

Evidently these are only moderately narrow states. As one might expect the final states $\mathrm{KK}, \mathrm{KK}^{*}$ and $\mathrm{K}^{*} \mathrm{~K}^{*}$ are important for $s \bar{s}$. The mode $\eta \phi$ is much more attractive, however, as we do not expect $n \bar{n}(n=u, d)$ mesons to couple significantly to this channel. This mode is in effect an " $s \bar{s}$ filter", and should be much more attractive for identifying $s \bar{s}$ states than the open-strange modes involving kaons.

Turning to open-strange mesons, we find that the "strangest" state in the known strange meson spectrum is the $\mathrm{K}^{*}(1414)$. First, the mass of this state appears much too light for a $2 \mathrm{~S}$ radial vector kaon, given the nonstrange candidates $\rho(1465)$ and $\omega(1419)$. It is also very surprising that it would have a lower mass than the radial pseudoscalar kaon $\mathrm{K}(1460)$. The decays of the $\mathrm{K}^{*}(1414)$ are also a problem; in the ${ }^{3} \mathrm{P}_{0}$ model the dominant mode is predicted to be $\pi \mathrm{K}$, with a branching fraction of about $30 \%$. Experimentally, the branching fraction observed by LASS was only $6 \%$. These discrepancies in mass and decays suggest a problem with a simple $2 \mathrm{~S}$ radial $\mathrm{K}^{*}$ assignment. One exciting possibility is that we may be seeing the effect of mixing with exotic vector hybrid states; since there is no C-parity in kaons, the $1^{--}$and $1^{-+}$basis states mix, giving an overpopulation of vector states and a different mass matrix for $1^{-}$kaons than for $1^{--} \rho$ and $\omega$ excited vectors. A simple comparison of strange and nonstrange excited vectors may therefore show the presence of the additional $1^{-+}$hybrid basis states, and with significant hybrid mixing we would expect the excited kaons to have rather different masses and decay amplitudes than their nonstrange partners.

There are also very interesting issues regarding the singlet-triplet mixing angles of excited kaons (analogous to the $\mathrm{K}_{1}(1273)-\mathrm{K}_{1}(1402)$ mixing angle), which can be determined from decay amplitudes of these excited states. Another interesting aspect of kaon strong decays is the relative strength of $\eta$ and $\eta^{\prime}$ modes, which depends on the angular quantum numbers in a complicated manner due to an interference between the $n \bar{n}$ and $s \bar{s}$ components of the $\eta$ and $\eta^{\prime}$ [17]. These selection rules also have applications to B decays involving an $\eta$ or $\eta^{\prime}$ [20]. 


\section{STRONG DECAYS OF EXOTICA}

\section{Hybrids}

The most interesting and influential predictions of meson decay models in recent years have been the predictions for the decay modes of exotica, specifically hybrid (excited glue) mesons. In the flux tube model of Isgur and Paton, hybrids are treated as states of quark, antiquark and flux-tube, in which the flux-tube is spatially excited about the quark-antiquark axis. When this simple quantum mechanical picture is combined with a ${ }^{3} \mathrm{P}_{0}$ model for pair production, which is assumed to take place along the path of the flux tube, one obtains a simple, intuitive picture of the open-flavor decay amplitudes of gluonic hybrid mesons.

Isgur, Paton and Kokoski [21] found that this simple picture gave a plausible explanation as to why the predicted rich spectrum of hybrids had not been clearly identified; the preferred decays to conventional two-meson final states showed a strong preference for the so-called "S+P modes", in which one of the final mesons had an internal orbital excitation. Since the P-wave mesons have secondary decays, a search for hybrids would require a study of complicated multimeson final states, which had not previously been considered systematically. In addition the numerical scales of the strong widths of hybrids were in many cases rather large, so that the hybrids would be difficult to identify. Isgur et al. cited the $\mathrm{I}=1$ exotic with $\mathrm{J}^{P C}=1^{-+}$as a case of special interest, since the total width of this state was found to be rather narrow, $\Gamma \approx 150 \mathrm{MeV}$, for an initial hybrid mass of $1.9 \mathrm{GeV}$. The preferred decay modes of this hybrid in the flux tube decay model were $b_{1} \pi$ and $f_{1} \pi$; this observation has stimulated several experimental studies of these rather complicated final states.

Isgur et al. only considered the decays of the $\mathrm{J}^{P C}$-exotic hybrids. These flux tube hybrid decay calculations were extended to nonexotic hybrids by Close and Page [22], who found that some very interesting and relatively narrow nonexotics should appear in the spectrum as an overpopulation relative to the naive quark model, provided that the flux tube picture of hybrids and their decays is reasonably accurate. Two notable cases are an extra $\omega$ (predicted to be only $100 \mathrm{MeV}$ wide, and to decay mainly to the $\mathrm{KK}_{1}$ channels) and an extra $\pi_{2}$. This nonexotic hybrid $\pi_{2}$ is predicted to have a very characteristic $b_{1} \pi$ mode; this mode is forbidden to the conventional $q \bar{q}$ quark model $\pi_{2}$ (presumably the $\pi_{2}(1670)$ ), because it is an $\left(S_{q \bar{q}}=0\right) \rightarrow\left(S_{q \bar{q}}=0\right)+\left(S_{q \bar{q}}=0\right)$ transition. These are forbidden for $(q \bar{q}) \rightarrow(q \bar{q})+(q \bar{q})$ in the ${ }^{3} \mathrm{P}_{0}$ model as well as in OGE pair production and linear scalar pair production models. In contrast they are allowed for decays of flux-tube hybrids, since the $\pi_{2}$ hybrid actually has the quarks in an $S_{q \bar{q}}=1$ configuration. The very strong experimental limit of the $b_{1} \pi$ mode of the $\pi_{2}(1670)$ reported by VES is implicitly a constraint on the size of the hybrid component of the $\pi_{2}(1670)$ state vector.

As a final topic in hybrid decays, there have been very interesting recent results from LGT on the closed-flavor decays of heavy hybrids. The UKQCD collaboration [23] has found that some of these modes, notably to a $\chi$ state and a scalar (presumably an effectively $\pi \pi$ ) are remarkably large. This result is very surprising in view of the weakness of 
the known closed-flavor $c \bar{c}$ and $b \bar{b}$ dipion decays, but if correct suggests very attractive modes for heavy hybrid searches, such as $(\gamma \mathrm{J} / \psi)+(\pi \pi)_{S}$ for charmonium hybrids.

\section{Glueballs}

The search for glueballs is a central component of the more general search for exotica in hadron spectroscopy. The well known LGT study of the glueball spectrum by Morningstar and Peardon [24] shows that the currently experimentally accessible glueballs have non-exotic quantum numbers, since the lightest predicted glueball exotic is a $2^{+-}$state at a very high mass of $\sim 4 \mathrm{GeV}$. At the presently accessible masses of up to ca. $2.5 \mathrm{GeV}$ just three glueballs are anticipated, a $0^{++}$near $1.6 \mathrm{GeV}$ and a $0^{-+}$and $2^{++}$near $2.3 \mathrm{GeV}$. The scalar channel is most interesting at present because we have two experimental candidates for this lightest glueball, the $f_{0}(1500)$ and $f_{0}(1710)$.

Although width and decay mode predictions for glueballs are obviously of paramount importance, there has been little work in this area. The naive expectation that glueballs should have flavor-blind decays is clearly strongly violated by both experimental candidates; the $f_{0}(1500)$ shows a strong preference for $\pi \pi$ modes over $\mathrm{KK}$, and the $f_{0}(1710)$ shows the inverse pattern. This has been attributed to strong mixing between the pure glue basis state and the scalar quarkonium $|n \bar{n}\rangle$ and $|s \bar{s}\rangle$ basis states, analogous to $\eta-\eta^{\prime}$ mixing in the $0^{-+}$sector (see for example the work of Amsler and Close [25]).

An alternative explanation for the violation of flavor-singlet decay symmetry in the scalar glueball candidates has been suggested by Sexton et al. [26, 27], based on a LGT study of the glueball-Ps-Ps three-point function. In this early lattice study they found a strong dependence of this glueball decay coupling on the mass assumed for the final pseudoscalar mesons, which if correct would skew mixing angle determinations using flavor-symmetric couplings, as assumed by Amsler and Close. The Sexton et al. LGT couplings favored the $f_{0}(1710)$ over the $f_{0}(1500)$ as a scalar glueball candidate.

The very important topic of glueball decay amplitudes merits more careful consideration in future LGT studies. The decay couplings the scalar glueball to vector meson pairs (which may be important for the $f_{0}(1500)$ ) and the couplings of the lightest $0^{-+}$ and $2^{++}$excited glueballs (as regards favored modes and total widths) are also important topics for future lattice studies.

\section{STUDIES OF THE UNDERLYING QCD DECAY MECHANISMS}

Strong decay amplitudes are crucial properties of hadrons, which if understood even at a phenomenological level can be used to identify plausible candidates for the various conventional quark model hadrons as well as the exotica predicted by model studies and lattice QCD.

Of course there is a deeper question, which is the problem of what fundamental interaction in QCD is responsible for hadron strong decays at the quark-gluon level. Although we can develop phenomenological models such as the ${ }^{3} \mathrm{P}_{0}$ model without understand- 
ing the decay mechanism, these models are simply approximate descriptions of decays with unknown and perhaps large systematic approximations. The QCD mechanism that drives the open-flavor strong decays we have discussed here is not well established, and has been studied in surprisingly few reference. Here we will discuss the results of two of these references, which reach rather similar general conclusions.

The original Cornell charmonium group perhaps surprisingly did not assume the ${ }^{3} \mathrm{P}_{0}$ decay model. Instead they assumed a nonperturbative microscopic model for strong decays, in which $q \bar{q}$ pair production took place through the linear confining interaction, treated as an exchange between the constituent $c$ and $\bar{c}$ quarks and the produced light $q \bar{q}$ pair (see Fig.2). This model is interesting in part because it is so highly constrained; the numerical value of the string tension is reasonably well known from spectroscopy, and therefore gives decay predictions that have no free parameters. This model gives rather good predictions for the behavior of $R$ (specifically charmonium production above open charm threshold), which implicitly involves width calculations for the higher vector resonances. (These resonances were not treated individually in the Cornell studies, instead the contributions to $R$ were determined implicitly using an effecting interaction containing decay loops.) Nonetheless the assumption of a vector confining interaction is controversial, and would presumably fail the D/S ratio test in decays such as $b_{1} \rightarrow \omega \pi$.

A more recent paper by Ackleh et al. [6] investigates various possible QCD mechanisms for strong decays. This reference concludes that the naive OGE pair production diagram in most cases gives a rather small contribution to the decay amplitude, which (as was assumed by the Cornell group) is instead dominated by pair production from the linear confining interaction. Unlike the Cornell model however, Ackleh et al. assume a scalar confining interaction, which gives a D/S ratio for $b_{1} \rightarrow \omega \pi$ that is close to experiment. Again the decay rates are known in this type of model in terms of the string tension; the simple linear scalar potential gives somewhat larger decay amplitudes than are observed experimentally.

\section{A NOVEL APPLICATION: V-PS SCATTERING FROM FSI}

Watson's theorem implies that final state interactions induce a phase factor of $e^{i \delta_{f}}$ in the decay amplitude of a resonance into the channel $f$. If there is only a single distinguishable channel, as in $\rho \rightarrow \pi \pi$, this phase is not observable. However in many decays a given final state spans several different channels, which are distinguished for example by internal angular momenta.

An example of this FSI effect was recently exploited by Nozar et al. [28] in a very interesting new "application" of strong decays. The decay $b_{1} \rightarrow \omega \pi$ is well known as a textbook example of a meson decay to more than one channel, since the $\omega \pi$ system can be in both $\mathrm{S}$-wave $\left({ }^{3} \mathrm{~S}_{1}\right)$ and $\mathrm{D}$-wave $\left({ }^{3} \mathrm{D}_{1}\right)$ states. The ratio of $\mathrm{D}$-wave to $\mathrm{S}$-wave amplitudes was very important historically in selecting the ${ }^{3} \mathrm{P}_{0}$ model as a realistic description of pair production quantum numbers in meson decays.

Since the S- and D-wave states have different scattering phase shifts, they will have different FSI phases; after rescattering the final $\omega \pi$ state will be of the form 


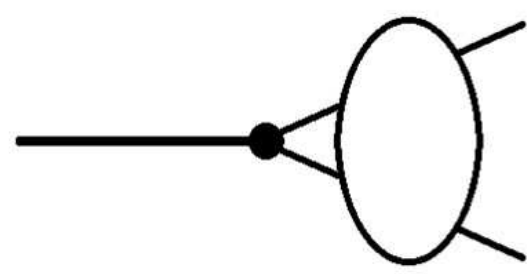

FIGURE 3. The meson decay FSI effect exploited by Nozar et al. [28] to study $\omega \pi$ scattering.

$$
|\omega \pi\rangle=a_{S} e^{i \delta_{S}}\left|\omega \pi\left({ }^{3} \mathrm{~S}_{1}\right)\right\rangle+a_{D} e^{i \delta_{D}}\left|\omega \pi\left({ }^{3} \mathrm{D}_{1}\right)\right\rangle,
$$

so the famous $\mathrm{D} / \mathrm{S}$ ratio is actually a complex number. The relative phase $\delta_{S}-\delta_{D}$ appears in the $a_{D} a_{S}$ cross term in the squared amplitude, and thus can be inferred directly from the observed $\omega \pi$ angular distribution. The study of $b_{1}$ decay can thus give us very exciting information on the scattering amplitudes of strongly unstable resonances, which would not otherwise be accessible.

This measurement was carried out by Nozar et al. [28], who found an $\omega \pi$ relative scattering phase at the $b_{1}$ mass of

$$
\left[\delta_{S}-\delta_{D}\right]_{\omega \pi}=-10.54^{\mathrm{o}} \pm 2.4^{\mathrm{o}}
$$

This is numerically rather similar to a quark model prediction of $\delta_{S}(\omega \pi)=-14^{\circ}$ by Barnes, Black and Swanson [29]. (One expects the $\omega \pi$ D-wave phase shift to be small at the $b_{1}$ mass.)

Clearly this is just one example of a large number of scattering amplitudes of strongly unstable resonances that can be inferred from high-statistics studies of strong decays, and future measurements should provide phase shifts that will be very interesting for theorists attempting to understand hadron scattering and FSI effects.

A caution is appropriate; we have assumed that the underlying decay amplitudes themselves are relatively real in different channels, and also that FSI effects are diagonal. Of course both these assumptions are suspect, and should be tested by comparing scattering phase shifts inferred from the decays of different initial mesons.

\section{FUTURE DECAYS: UNQUENCHING THE QUARK MODEL}

One of the exciting new discoveries [30] discussed at this meeting was the observation of the charm-strange mesons $\mathrm{D}_{S J}^{*}(2317)$ and $\mathrm{D}_{S J}^{*}(2357)$, which are presumably $0^{+}$and $1^{+}$ states respectively. The masses of these states are quite surprising, since the normally accurate potential model of Godfrey and Isgur [14] predicts much higher masses of $2.48 \mathrm{GeV}$ and $2.55 \mathrm{GeV}$ respectively. 
Since these states are predicted by the ${ }^{3} \mathrm{P}_{0}$ model to be very broad [31] and are quite close to DK threshold, one possibility is that the usual neglect of decay couplings in quark model calculations is inaccurate here, and the states have been displaced downwards by ca. $150 \mathrm{MeV}$ due to decay loops. This may imply that the states have large $\mathrm{DK}$ and $\mathrm{DK}^{*}$ molecular components respectively [32], rather like the $\mathrm{KK}$ molecules, instead of being simple $c \bar{s}$ quark model states.

This topic of the contribution of virtual decay loop diagrams to hadron properties, known as "unquenching the quark model", is an important but rather obscure issue. Explicit evaluation typically finds that individual loop diagrams are large, but that there may be significant cancellations (see for example [33]). Future studies of strong decays will undoubtedly include investigations of the effects of these virtual decay loops, since the $\mathrm{D}_{S J}^{*}(2317)$ and $\mathrm{D}_{s J}^{*}(2357)$ may have "announced" to us that in some strongly-coupled channels these effects cannot be ignored.

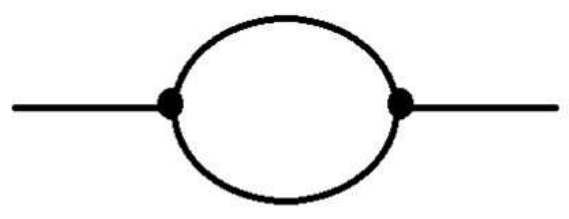

FIGURE 4. The generic loop diagram representing the second-order effects of virtual decay of a meson into the two-meson continuum.

\section{ACKNOWLEDGMENTS}

I would like to thank the organizers of HADRON03 for their kind invitation to present this summary of the history, current status and possible future of research in hadron strong decays. This research was supported in part by the U.S. National Science Foundation through grant NSF-PHY-0244786 at the University of Tennessee, and by the U.S. Department of Energy under contract DE-AC05-00OR22725 at Oak Ridge National Laboratory (ORNL).

\section{REFERENCES}

1. L.Micu, Nucl. Phys. B, 10, 521 (1969).

2. A.LeYaouanc, L.Oliver, O.Péne and J.C.Raynal, Phys. Rev. D, 8, 2223 (1973).

3. ibid., Phys. Rev. D, 9, 1415 (1974).

4. ibid., Phys. Rev. D, 11, 1272 (1975).

5. ibid., Phys. Lett. B, 71, 397 (1977).

6. E.S.Ackleh, T.Barnes and E.S.Swanson, Phys. Rev. D, 54, 6811 (1996).

7. E.Eichten, K.Gottfried, T.Kinoshita, K.D.Lane and T.-M.Yan, Phys. Rev. Lett., 36, 500 (1976).

8. ibid., Phys. Rev. D, 17, 3090 (1978).

9. ibid., Phys. Rev. D, 21, 203 (1980). 
10. E.S.Swanson, Nucl. Phys. Proc. Suppl., 64, 312 (1998).

11. R.M.Baltrusaitis et al., Phys. Rev. Lett., 56, 107 (1986).

12. S.Godfrey, R.Kokoski and N.Isgur, Phys. Lett. B, 141, 439 (1984).

13. H.G.Blundell and S.Godfrey, Phys. Rev. D, 53, 3700 (1996).

14. S.Godfrey and N.Isgur, Phys. Rev. D, 32, 189 (1985).

15. S.Capstick and N.Isgur, Phys. Rev. D, 34, 2809 (1986).

16. T.Barnes, F.E.Close, P.R.Page and E.S.Swanson, Phys. Rev. D, 55, 4157 (1997).

17. T.Barnes, N.Black and P.R.Page, Phys. Rev. D, 68, 054014 (2003).

18. S.Capstick and W.Roberts, Phys. Rev. D, 49, 4570 (1994).

19. ibid., Prog. Part. Nucl. Phys., 45, S241 (2000).

20. H.J.Lipkin, Phys. Lett. B, 494, 248 (2000).

21. N.Isgur, R.Kokoski and J.Paton, Phys. Rev. Lett., 54, 869 (1985).

22. F.E.Close and P.R.Page, Nucl. Phys. B, 443, 233 (1995).

23. C.McNeile, C.Michael and P.Pennanen (UKQCD Collaboration), Phys. Rev. D, 65, 094505 (2002).

24. C.J.Morningstar and M.Peardon, Phys. Rev. D, 60, 034509 (1999).

25. C.Amsler and F.E.Close, Phys. Rev. D, 53, 295 (1996).

26. J.Sexton, A.Vaccarino and D.Weingarten, Nucl. Phys. B, 42, 279 (1995).

27. J.Sexton, A. Vaccarino and D.Weingarten, Phys. Rev. Lett., 75, 4563 (1995).

28. M.Nozar et al. (E852 Collaboration), Phys. Lett. B, 541, 35 (2002).

29. T.Barnes, N.Black and E.S.Swanson, Phys. Rev. C, 63, 025204 (2001).

30. B.Aubert et al. (BABAR Collaboration), Phys. Rev. Lett., 90, 242001 (2003).

31. S.Godfrey and R.Kokoski, Phys. Rev. D, 43, 1679 (1991).

32. T.Barnes, F.E.Close and H.J.Lipkin, Phys. Rev. D, 68, 054006 (2003).

33. P.Geiger and N.Isgur, Phys. Rev. Lett., 67, 1066 (1991). 